Patentregister

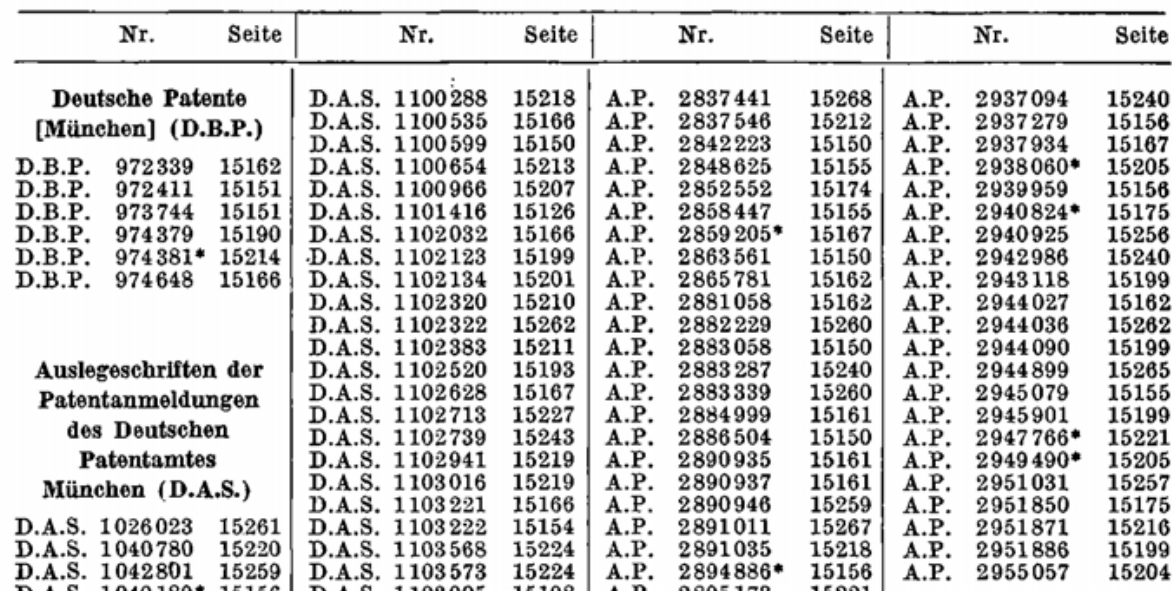

D.A.S. $1042801 \quad 15259$

D.A.S. $1049189 * 15156$

D.A.S. 105802615151

D.A.S. 1060567 * 15167

D.A.S. $1067817 \quad 15204$

D.A.S. 106958215151

D.A.S. $1071080 \quad 15125$

D.A.S. $1072240 \quad 15202$

D.A.S. 107721015197

D.A.S. 107764215151

D.A.S. 107857015201

D.A.S. 108099515197

D.A.S. $1081007 \quad 15200$

D.A.S. $1083231 \quad 15159$

D.A.S. $1083650 \quad 15268$

D.A.S. 1083824 15124

D.A.S. 1085087 * 15167

D.A.S. $1086124 \quad 15268$

D.A.S. $1086376 \quad 15258$

D.A.S. 1089077 * 15157

D.A.S. $1089391 \quad 15200$

D.A.S. 109065915201

D.A.S. $1090788 \quad 15150$

D.A.S. 1091161* 15156

D.A.S. $1091396 \quad 15193$

D.A.S. $1091750 \quad 15219$

D.A.S. 1092078* 15157

D.A.S. 1092926* 15205

D.A.S. 109344315156

D.A.S. 109373915160

D.A.S. $1094390 \quad 15258$

D.A.S. 1094727 * 15205

D.A.S. 1094828* 15157

D.A.S. $1095115 \quad 15267$

D.A.S. 1095335 15155

D.A.S. 109543015212

D.A.S. 1095976* 15244

D.A.S. 109629515159

D.A.S. $1096353 \quad 15126$

D.A.S. 1096438* 15157

D.A.S. 109652515264

D.A.S. $1096917 \quad 1520 ?$

D.A.S. $1097274 \quad 15247$

D.A.S. $1097405 \quad 15149$

D.A.S. $1097421 * 15163$

D.A.S. $1097427 \quad 15200$

D.A.S. $1097433 \quad 15198$

D.A.S. $1097440 \quad 15126$

D.A.S. 109820015220

D.A.S. 109850615196

D.A.S. $1098652 \quad 15209$

D.A.S. 109865515263

D.A.S. 109865615263

D.A.S. $1099111 \quad 15263$

D.A.S. 109912815227

D.AS. 1099164

15219

D.A.S. 1099675

Deutsche Patente (D.P.[DDR])

D.P.[DDR]

D.P.[DDR] 15155

$\begin{array}{rr}18047 & 15200\end{array}$

D.P.[DDR $18828 \quad 15154$

P. 19

15247

D.P.[DDR]

D.P.[DDR

D.P.[DDR]

D.P.[DDR]

$20511 \quad 15262$

$20794 \quad 15250$

Amerikanische Patente (A.P.)

A.P. 2734244

A.P. 2751378

A.P. 2754199 *

A.P. 2764480 .

A.P. 2783991 *

P. 2785973

A.P. 2793107

A.P. 2794732 *

A.P. 2798067

A.P. $2806786^{\circ}$

2812321

A.P. 2816060

A.P. $\quad 2819229$

A.P. 2820728 .

A.P. 2833801

\begin{tabular}{ll|ll} 
D.A.S. 1099738 & 15216 & A.P. & $\mathbf{2 8 3 4 7 0 6}$ \\
D.A.S. 1100209 & $\mathbf{1 5 2 1 0}$ & A.P. & $\mathbf{2 8 3 5 5 8 1}$
\end{tabular}
15150

15154

15121

15154

15127

15175

P. 2894886

A.P. $2895985^{\circ}$

5126

15194

15194

15194

15192

15194

15266

15156

15209

15120
15258

15214

15156

15221

15175
15266

A.P. 2932566 ,
A.P. 2897183

A.P. 2898291

A.P. 2898377

A.P. 2901326

A.P. 2902335

A.P. $\quad 2903339$

A.P. 2903358

A.P. 2903386

A.P. $\quad 2907073$

A.P. 2907682

A.P. 2912301

A.P. 2912321

A.P. 2912337

A.P. 2912340 .

A.P. 2913325

A.P. 2913326

A.P. 2913328

A.P. 2913340

A.P. 2913417

A.P. 2914072

A.P. $\quad 2914477$

A.P. 2917369

A.P. $\quad 2919209$

A.P. 2920032

A.P. $29: 1015$

A.P. 2921037

A.P. 2924570

A.P. 2925336

A.P. 2925338

A.P. 2926096

A.P. 2926105

A.P. 2926131

A.P. 2927034

A.P. 2927037

A.P. $\quad 2928861$

A.P. $\quad 2929772$

A.P. 2929813

A.P. 2930673

A.P. 2930714

15205

15259

15196

15161

15251

15161

15240

15192

15261

15264

15190

15240

15167

15174

15175

15240

15243

15240

15260

5161

15256

15258

15220

15257

15190

15267
15246

15246

1526

1525

15251

15199

15198

15162

15262

A.P. 2930749 , 1525

A.P. $2930807^{\circ}$

A.P. 2931788

A.P. 2931823

A.P. $\quad 2931829$

A.P. 2931831

A.P. 2932611

A.P. 2933464*

A.P. 2934497

A.P. $\quad 2935467$

A.P. 2935478 .

A.P. 2935501

A.P. 2935530 *

A.P. 2936216

A.P. 2936372
Australische Patente (Aust.P.)

Aust.P. $161189 * 15163$

Aust.P. 161 191* 15163

Aust.P. $161551 \quad 15257$

Aust.P. 161959* 15175

Aust.P. 209400* 15151

Aust.P. $215043 \quad 15217$

Aust.P. 218452* 15205

Aust.P. 218772* 15193

Aust.P. 218779* 15193

Aust.P. $219508 \quad 15149$

Aust.P. 219571 15155

Aust.P. 220793 15167

Aust.P. $221527 \quad 15212$

Aust.P. $221537 \quad 15167$

\section{Belgische Patente}

(Belg.P.)

Belg.P. 517293* 15194 Belg.P. 533301* 15221 Belg.P. 541222 15260 Belg.P. 543003* 15128 Belg.P. $543929 \quad 15151$ Belg.P. 553949* 15222 Belg.P. 554475* 15221 Belg.P. 554943* 15128 Belg.P. 555031* 15205 Belg.P. 555319 15127 Belg.P. 555321 15127 Belg.P. 555531 15252 Belg.P. 556920* 15128 Belg.P. 556977* 15221 Belg.P. 558922 15190 Belg.P. $559288 \quad 15218$ Belg.P. 559894 15246 Belg.P. 560440* 15204 Belg.P. $561056 \quad 15174$ Belg.P. $561613 \quad 15209$ Belg.P. 561966 15263 Belg.P. 562538 15200 Belg.P. 562727* 15221. Belg.P. 562991* 15163 Belg.P. 563013 15127 Belg.P. $563014 \quad 15127$ Belg.P. $563015 \quad 15127$ Belg.P. 563016 15127 Belg.P. 564813* 15128 Belg.P. 565320* 15194 Belg.P. 565 449* 15193 Belg.P. 565698* 15193 Belg.P. 565 722* 15194 15156 Belg.P. 565823* 15128 


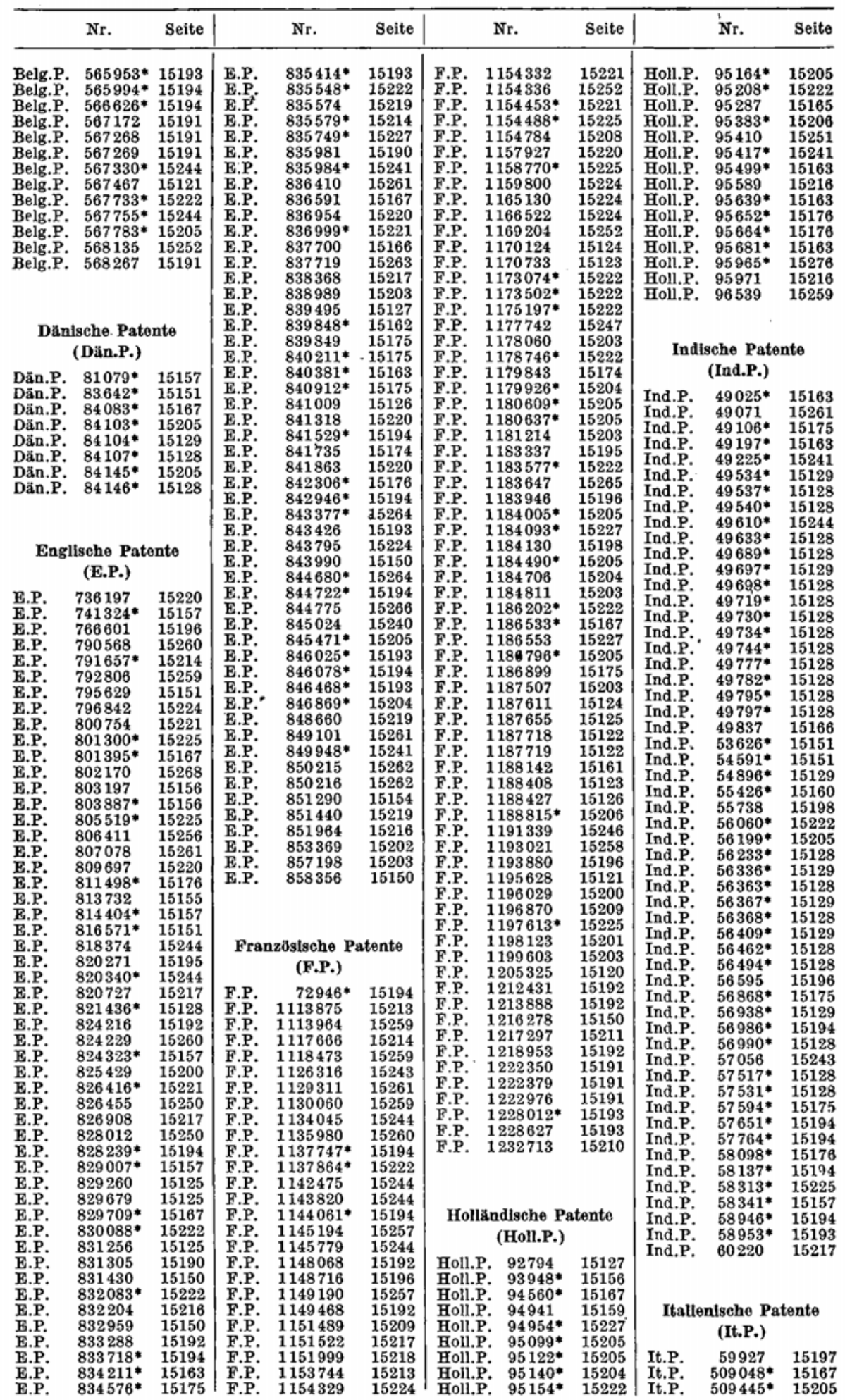




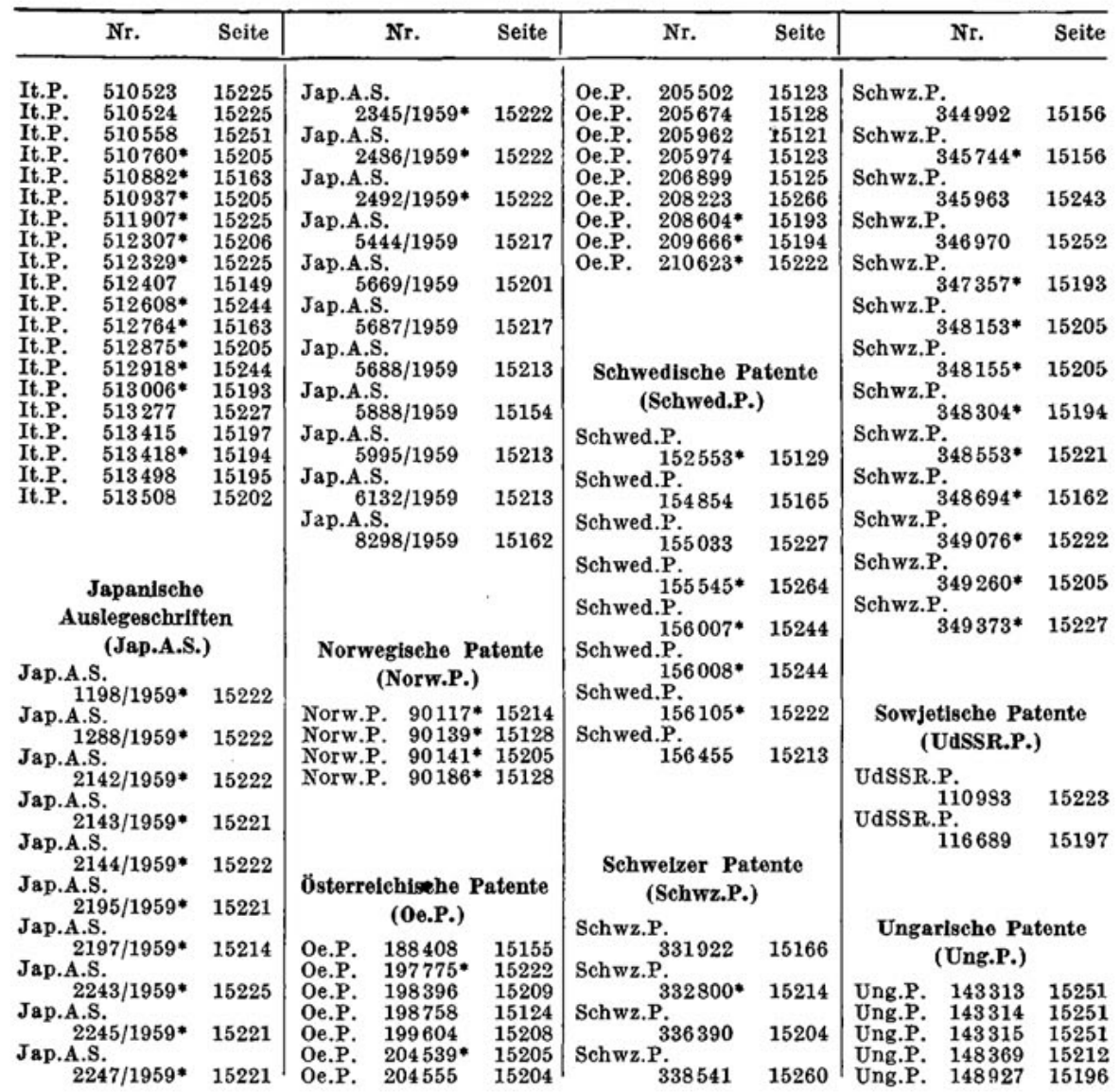

\section{J. C. POG GENDORFF}

\section{Biographisch-literarisches Handwörterbuch der exakten Naturwissensehaften}

Band VII a, Teil 4: S-Z, Fünfte Lieferung

Unter Mitwirkung

der Akademien der Wissenschaften zu Berlin, Göttingen,

Heidelberg, München und Wien

herausgegeben

von der Sächsischen Akademie der Wissenschaften zu Leipzig

Redigiert von Prof. Dr. Rudolph Zaunick

und Prof. Dr. Hans Salié

1961. 112 Seiten - gr. $8^{\circ}$ - DM 16, -

Bestellungen durch eine Buchhandlung erbeten

A K A D E M I E - V E R LA G • B E R L I N 